Tropical Journal of Pharmaceutical Research May 2019; 18 (5): 1069-1076

ISSN: $1596-5996$ (print); 1596-9827 (electronic)

(c) Pharmacotherapy Group, Faculty of Pharmacy, University of Benin, Benin City, 300001 Nigeria.

Available online at http://www.tjpr.org

Original Research Article

http://dx.doi.org/10.4314/tjpr.v18i5.22

\title{
Effect of bone morphogenetic protein-2 on diabetic retinopathy and its mechanism of action
}

\section{Zaohe Sun, Guangming Wan*, Shenzhi Liang, Cheng Qian}

Department of Ophthalmology, The First Affiliated Hospital of Zhengzhou University, Zhengzhou, Henan 450052, PR China

*For correspondence: Email: hnzz20080101@163.com

Sent for review: 22 January 2019

Revised accepted: 28 April 2019

\begin{abstract}
Purpose: To investigate the effect of bone morphogenetic protein-2 (BMP-2) on human retinal vascular endothelial cells (RECs) and human retinal pigment epithelial cells (RPE) cultured in high glucose (HG) in vitro, and the underlying mechanism.

Methods: Cell counting kit-8 (CCK-8) was used to determine cell proliferation while Western blot was used to assay the expressions of extracellular matrix and angiogenesis-related factors, Expressions of cytokines and chemokines were assessed by quantitative real time polymerase chain reaction (qRT$P C R$ ) and enzyme-linked immunosorbent assay (ELISA). Changes in Smad, ERK, JNK and p38MAPK signal pathway were measured by transfection and interference.

Results: The level of expression of BMP-2 in HG group was higher than that in normal glucose (NG) culture group. The expressions of angiogenesis-related factors i.e. vascular endothelial growth factor (VEGF) and intercellular cell adhesion molecule-1 (ICAM1), pro-inflammatory factors i.e. IL-6 and chemokine monocyte chemokine protein-1 (MCP1), increased significantly in HG group compared to $N G$ and HG + BMP-2 groups. Phosphorylation of Smad1/5/8 and activation of ERK, JNK and p38MAPK signaling pathways were enhanced by BMP-2.

Conclusion: These results suggest that BMP-2 promotes angiogenesis and enhances the expressions of inflammatory cytokines via Smad signaling pathway.
\end{abstract}

Keywords: Diabetic retinopathy, Retina, Pigment epithelial cells, Vascular endothelial cells, Bone morphogenetic protein-2

\begin{abstract}
This is an Open Access article that uses a fund-ing model which does not charge readers or their institutions for access and distributed under the terms of the Creative Commons Attribution License (http://creativecommons.org/licenses/by/4.0) and the Budapest Open Access Initiative (http://www.budapestopenaccessinitiative.org/read), which permit unrestricted use, distribution, and reproduction in any medium, provided the original work is properly credited.
\end{abstract}

Tropical Journal of Pharmaceutical Research is indexed by Science Citation Index (SciSearch), Scopus, International Pharmaceutical Abstract, Chemical Abstracts, Embase, Index Copernicus, EBSCO, African Index Medicus, JournalSeek, Journal Citation Reports/Science Edition, Directory of Open Access Journals (DOAJ), African Journal Online, Bioline International, Open-J-Gate and Pharmacy Abstracts

\section{INTRODUCTION}

Diabetic retinopathy (DR) is one of the predisposing factors to blindness among people in the age range of $26-75$ years. It is also a major socio-economic burden in most countries [1]. The most important markers of vascular injury during DR are leukocytosis, high permeability of blood vessels, and early-stage inflammatory response, followed by retinal neovascularization (RNV) [2]. Diabetic retinopathy-induced vision loss is due to the rupture of the blood-retinal barrier (BRB) which results in macular edema, retinal detachment, and intra-retinal and vitreous hemorrhage. Several growth factors have been shown to play 
vital roles in the progression of DR. These are vascular endothelial growth factor (VEGF), angiopoietin, and insulin-like growth factor [3]. However, the potential molecular mechanisms involved in DR have not yet been fully understood.

Bone morphogenetic protein (BMP) contains a wide range of conserved growth factor groups, more than 30 members of which have been identified to date, and it is the largest member of the transforming growth factor beta (TGF $\beta$ ) superfamily [4]. Three members of this family i.e. BMP-2, BMP-4 and BMP-7 and their receptors (BMPR) play important roles in ocular biochemistry. Some researchers have suggested that BMP signaling may be involved in regulation of retinal vascular homeostasis and diabetesinduced vascular dysfunction such as atherosclerosis [5]. The BMP signaling pathway is involved in the regulation of endothelial cell tube formation and oxidative stress during angiogenesis. The association between BMP and angiogenesis was demonstrated by the discovery of BMPER, a precursor of BMP in endothelial cells, indicating that BMP-2 is an extracellular regulator needed for normal BMP signaling [6]. Recently, the role of BMPER in regulating protein levels after angiogenesis stimulation in oxygen-induced retinopathy (OIR) mouse models has been reported [7].

However, in spite of the fact that these evidence suggest that BMP plays a key role in the induction and maintenance of vascular inflammation and angiogenesis in DR, the underlying mechanisms remain relatively unknown. In addition, the function of BMP-2 in the pathogenesis of DR is not clear. Therefore, in the present study, the effect BMP-2 on retinal pigment epithelium (RPE) and retinal vascular endothelial cells (RECs) cultured in HG, and the mechanisms involved, were investigated in vitro. This was with a view to providing new scientific information that might enhance the prevention and treatment of DR.

\section{EXPERIMENTAL}

\section{CCK8 cell viability assay}

Cells were inoculated in 96-well plates at a density of $5 \times 10^{3}$ cells $/ \mathrm{mL}$. The plate was cultured at $37 \circ \mathrm{C}$ in a humidified incubator containing $5 \% \mathrm{CO}_{2}$. After cell fusion, RECs cells were divided into three groups: NG culture group, HG group, and high glucose + BMP-2 group. Then, $10 \mu \mathrm{L}$ CCK- 8 solution was mixed and added to each well in the 96-well culture plate and incubated for 1 hour. Absorbance was determined at $450 \mathrm{~nm}$ in an ELISA monitor, and the results were recorded.

\section{Western blotting}

The different cell groups were harvested with centrifugation and lysed in protease-inhibitor containing lysis buffer. A portion of total protein from each lysate was subjected to SDS-PAGE, followed by transference to polyvinylidene difluoride (PVDF) membrane. Non-specific binding was blocked by incubating the membranes with non-fat milk for $1 \mathrm{~h}$ at room temperature. The PVDF membranes were thereafter incubated overnight with the appropriate primary antibodies at $4{ }^{\circ} \mathrm{C}$. Then, incubation with horseradish-conjugated secondary antibody was carried out. The level of expressions of the various proteins were determined using infrared imaging. Protein expressions were normalized to that of $\beta$-actin which was used as control.

\section{Real-time PCR analysis}

After RNA extraction and CDNA reverse transcription, RT-PCR was performed. Repeated denaturation and annealing were extended for 40 cycles for amplification [8]. Finally, the threshold cycle (CT) values and the melting curve parameters were calculated, and the levels of expression were estimated using the $2^{-\Delta \Delta C T}$ method.

\section{Statistical analysis}

Data are expressed as mean \pm standard deviation (SD). One-way ANOVA was used for comparison between groups, while Least Significant Difference (LSD) was used for multiple comparisons. All statistical analyses were done with SPSS version 13.0. Values of $p<$ 0.05 were considered statistically significant.

\section{RESULTS}

High glucose induced expression and secretion of BMP-2 in hREC and ARPE-19 cells

The results in Figure I show that BMP-2 expression was significantly increased in the $\mathrm{HG}$ group $(p \leq 0.05)$.

\section{BMP-2 promoted hREC cell proliferation under high glucose conditions}

As shown in Figure 2, cell viability in the $\mathrm{HG}$ group was significantly lower than that in the NG group. Cell viability increased gradually in 
response to increases in the concentration of BMP-2. The cell viability of $\mathrm{HG}+\mathrm{BMP}-2$ group was significantly higher than that of the $\mathrm{HG}$ group $(p<0.05)$.
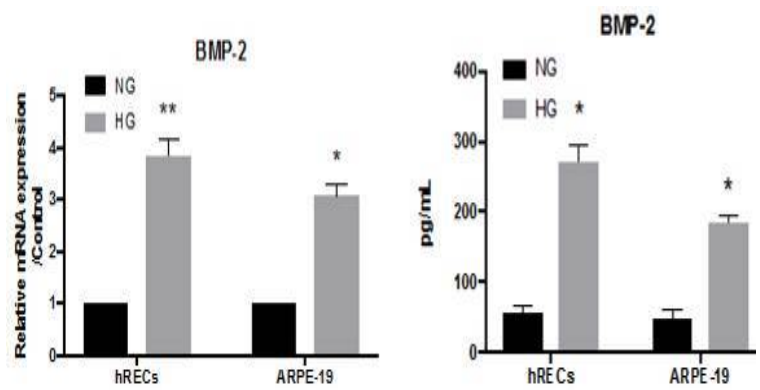

Figure 1: Effect of high glucose on the expression of BMP-2 in the cells

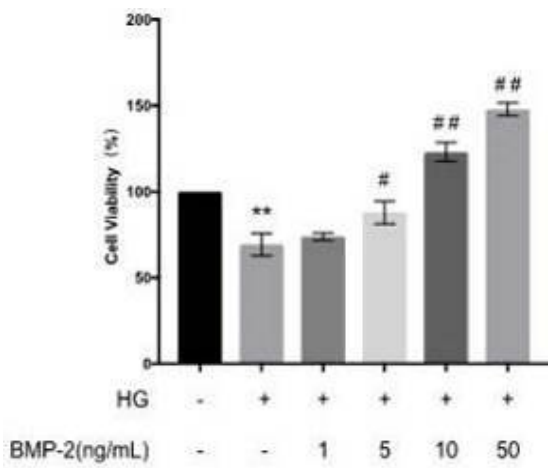

Figure 2: Effect of high glucose and BMP-2 treatments on cell viability

BMP-2 increased the expressions of angiogenic factors in hREC cells under high glucose conditions

As shown in Figure 3, the expressions of VEGF and ICAM-1 in the HG group were significantly higher than those in the control group, and the expressions of VEGF and ICAM- 1 in the HG + BMP-2 group were markedly higher than those in the pure HG group $(p<0.05)$.

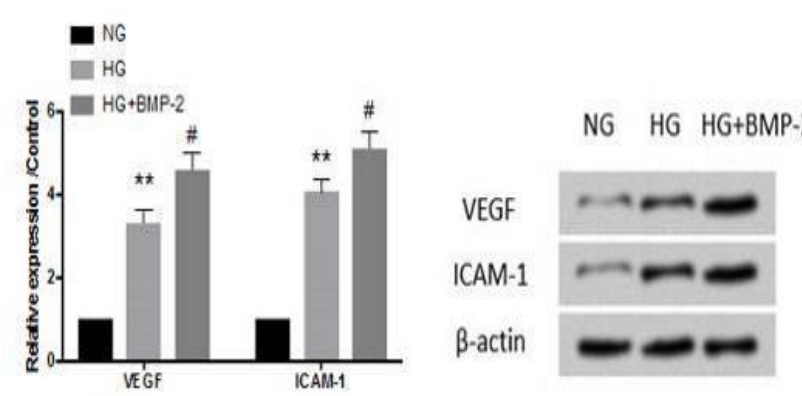

Figure 3: Effect of high glucose and BMP-2 treatments on expressions of VEGF and ICAM-1
BMP-2 inhibited the expression of ZO-1 in the extracellular matrix of ARPE-19 cells and promoted the expression of SMA $\alpha$ and MMP2

Figure 4 shows that the expression of cell ZO-1 in the $\mathrm{HG}$ group was significantly lower than that in the NG group. Moreover, the expression of $\mathrm{ZO}-1$ in $\mathrm{HG}+\mathrm{BMP}-2$ group was lower than that in $\mathrm{HG}$ group. These results demonstrated that the migration ability in the HG + BMP-2 group was lower than that in the pure $\mathrm{HG}$ group $(p<$ 0.05 ). In contrast, the expressions of SMA $\alpha$ and MMP2 in HG group was markedly higher than that in NG group, and the expression of $\mathrm{HG}+$ BMP-2 group was significantly higher than that in HG group $(p<0.05)$. These results indicate that the expression of ZO-1 was inhibited by BMP-2, while the expressions of SMA $\alpha$ and MMP2 were enhanced by BMP-2.

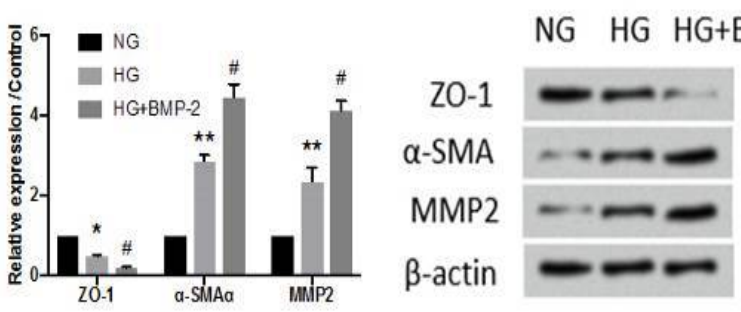

Figure 4: Changes in extracellular matrix proteins after high glucose andBMP-2 treatment

BMP-2 enhanced the expression of ARPE-19 cytokine in hyperglycemia

Figure 5 shows that the expression of cytoangiogenic factor VEGF in the HG group was markedly higher than that in the NG group. Moreover, the expression of VEGF in the $\mathrm{HG}+$ BMP-2 group was remarkably higher than that in the simple HG group $(p<0.05)$. These results suggest that the expression and secretion of angiogenesis factor VEGF were enhanced by BMP-2.

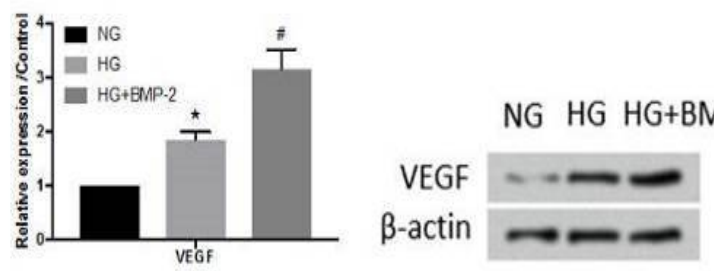

Figure 5: Effect of high glucose and BMP-2 treatment on the expression of ARPE-19

BMP-2 increased the expressions of hREC and ARPE-19 under high glucose

Figure 6 shows that the expressions of pro- 
inflammatory cytokines IL-6 and MCP-1 in the $H G$ group were significantly higher than those in the NG group. Moreover, the expressions of IL-6 and MCP-1 in the HG + BMP-2 group were significantly higher than those in the simple HG group $(p<0.05)$.

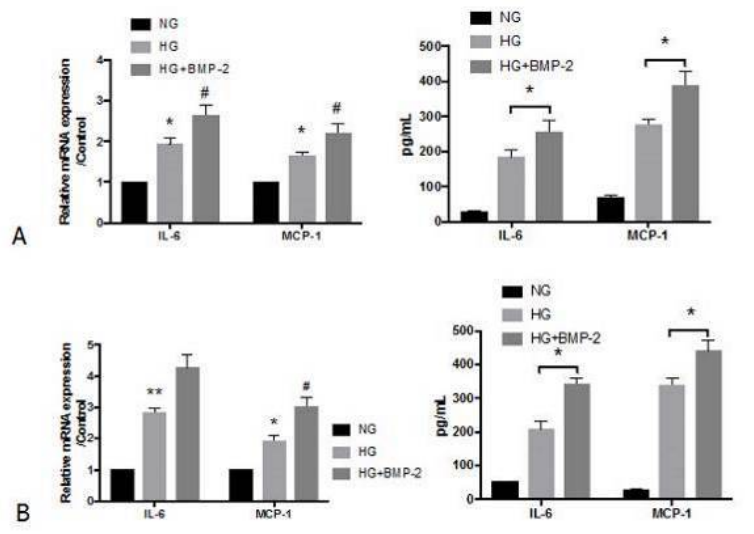

Figure 6: Effect of high glucose and BMP-2 treatment on expression levels of pro-inflammatory factors. A: hREC cell; B: ARPE-19 cell

BMP-2 influenced the expressions of angiogenesis and inflammatory factor hRECs through Smad-dependent and non-smaddependent pathways, while BMP-2 influenced angiogenesis and inflammatory factor expression in ARPE-19 cells through Smad-dependent pathways
The results of Western blot are shown in Figure 7 and Figure 8. The expression levels of Smad, ERK, JNK and MAPK in the HG group were significantly higher than the corresponding expression levels in the NG group $(p<0.05)$. However, the expressions of these proteins were significantly lower than those in the HG + BMP-2 group in both hRECs and ARPE-19 cells $(p<$ $0.05)$. These results suggest that BMP-2 promoted the phosphorylation of smad $1 / 5 / 8$ and the activation of ERK, JNK and p38MAPK signaling pathway in both hRECs and ARPE-19 cells (Figure $7 \mathrm{~A}$ and $\mathrm{B}$, Figure $8 \mathrm{~A}$ and $\mathrm{B}$ ).

With Smad protein knockout, there were significant reductions in the expressions of proangiogenic factors and inflammatory factors. However, in hRECs cells, the effect of BMP-2 was not completely offset. These results demonstrate that BMP-2 influenced angiogenesis and expression of inflammatory factors through a mechanism that is partially dependent on the Smad signaling pathway in hRECs cells (Figure $7 \mathrm{C}$ to E). In contrast, knockout of Smad protein markedly decreased the expressions of angiogenic factors and inflammatory factors in ARPE-19 cells, but there was no significant difference between $H G+B M P-2$ group and $H G$ group. Thus, BMP-2 affected angiogenesis and inflammatory factor expression in ARPE-19 cells through a mechanism that completely depends on the Smad signaling pathway (Figure $8 \mathrm{C}$ to $\mathrm{E}$ ).
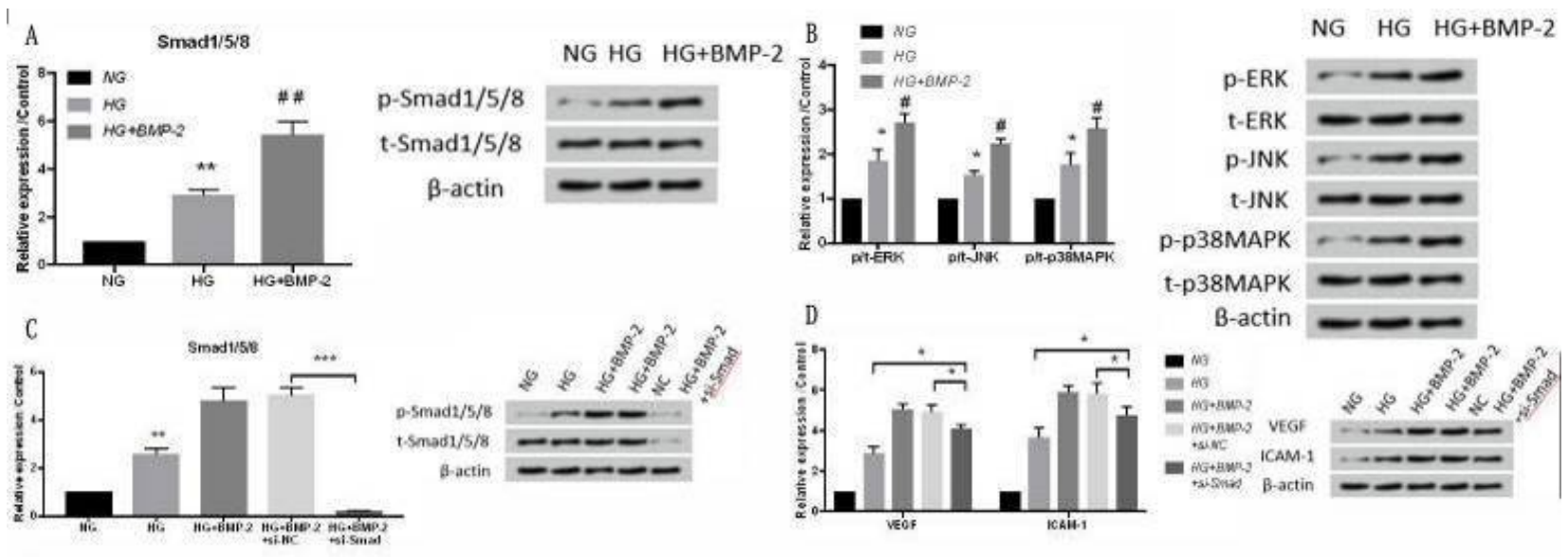

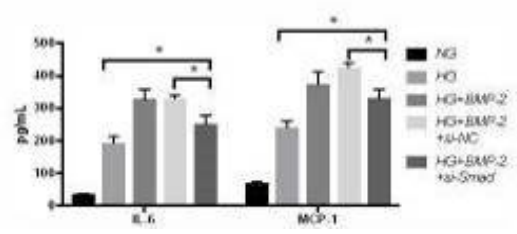

Figure 7: Changes in hREC cell signaling pathway proteins after high glucose and BMP-2 treatments 
A
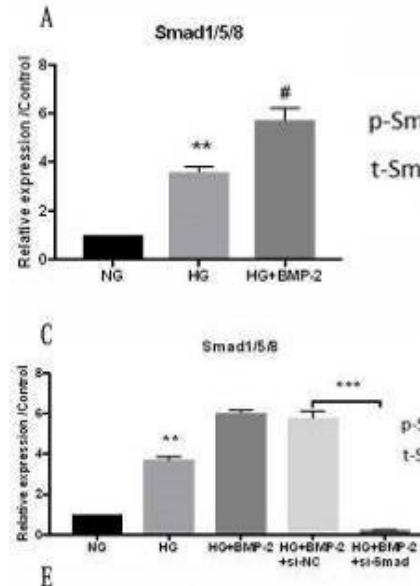

E

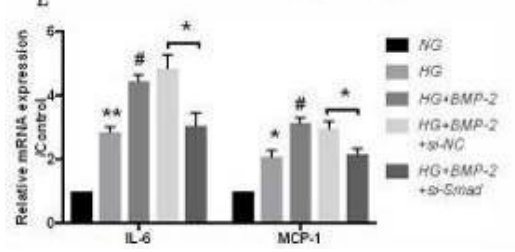

B
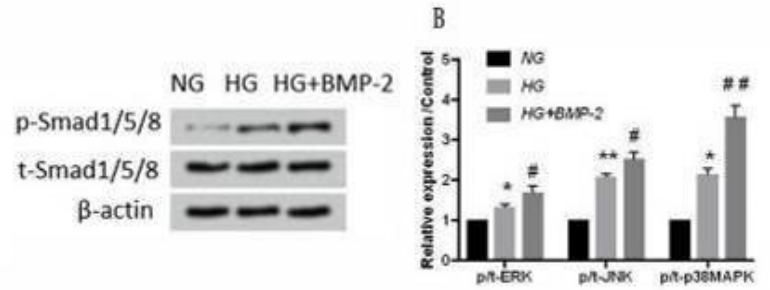

D

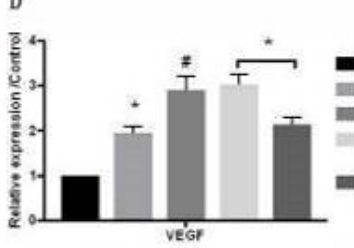

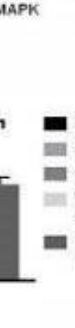

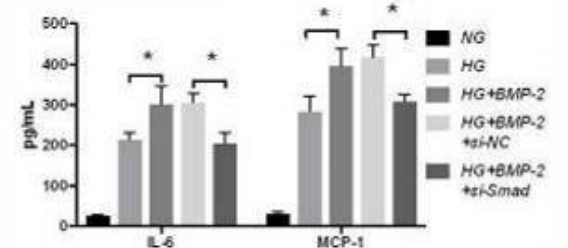

NG HG HG+BMP-2

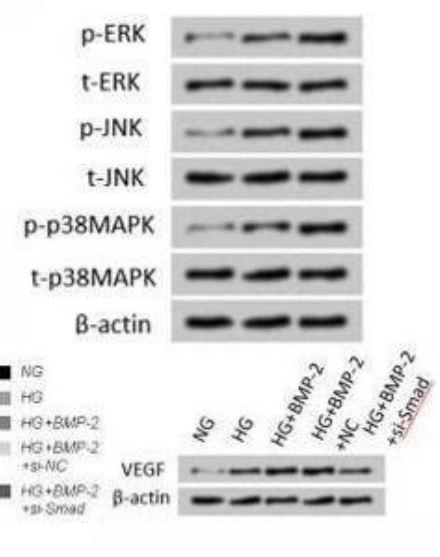

Figure 8: Effect of high glucose and BMP-2 treatments on ARPE-19 signaling pathway proteins

\section{DISCUSSION}

Diabetic retinopathy (DR) is a serious complication of diabetes mellitus (DM) and a major cause of visual impairment and blindness in adults [9].The clinical diagnosis of DR is based on abnormal performance of retinal vascular lesions. Accordingly, DR is divided into two stages: non-proliferative diabetic retinopathy (NPDR) and proliferative diabetic retinopathy (PDR) [10].

The most common cause of vision loss in DR patients is diabetic macular edema (DME) [11]. It appears at any stage of DR, resulting in loss of vision or distortion of visual images. The current specific treatment strategies for DR include intravitreal drug therapy, laser photocoagulation and vitreous surgery, all of which are aimed at managing microvascular complications [12]. At present, the primary treatment strategy for any stage of DR is the application of anti-VEGF drugs. Traditional laser treatment may partially enhance stable vision, while anti-VEGF treatment can improve vision and reduce adverse eye reactions. The poor therapeutic effect of anti-VEGF may be due to the participation of other VEGF-related molecular signaling pathways in its pathogenesis. Thus, it is important to investigate the potential mechanisms of DR and provide a new insight for the development of new methods of DR treatment.

It has been reported in vivo and in vitro that high glucose induces apoptosis of pericytes $[13,14]$.
Since pericytes are responsible for providing the supporting structure of capillaries, the loss of capillary walls can lead to local exudation of capillaries. This process is linked to the formation of microaneurysm which appears as an initial clinical symptom of DR. Furthermore, pathological changes such as decreases in pericytes, apoptosis of endothelial cells and thickening of basement membrane manifest in the pathogenesis of DR and blood-retina barrier (BRP) damage [15]. Studies have shown that the regulation of leukocyte migration and activation of chemokines are associated with the pathogenesis of DR [16]. It has been reported that the levels of chemokines secreted by macrophages such as monocyte inflammatory

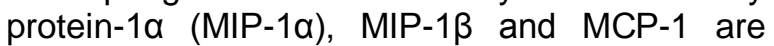
significantly increased in diabetic patients. In diabetic mice, it has been shown that MCP-1 deficiency might result in decreased retinal vascular leakage [17]. In addition, the expressions of serum inflammatory factors, for example tumor necrosis factor $\alpha$ (TNF- $\alpha$ ), interleukin 6 (IL-6), IL-8 and IL-1 $\beta$ are significantly upregulated in diabetic patients, and it has been demonstrated that the increased levels reflect the severity of DR [18].

Microglias are activated by high-glucose stress, which results in increased secretions of TNF- $\alpha$, IL-6, MCP-1, and VEGF. Consistent with the results of this study, the expressions of the angiogenesis-related factors VEGF and ICAM1 in the $H G$ group were significantly higher than those in the NG culture group. Moreover, the expression levels of angiogenesis-related factors 
in $\mathrm{HG}+$ BMP-2 group were higher than their corresponding expression levels in the $\mathrm{HG}$ group. Results from qRT-PCR and ELISA showed that the expression levels of the proinflammatory cytokine IL-6 and the chemokine MCP1 in HG group were significantly higher than those in NG group, but were lower than those in the $\mathrm{HG}+\mathrm{BMP}-2$ group.

Retinal neurodegeneration is an initial stage in the development of DR [19]. In animal models, apoptosis of retinal nerve cells manifest in diabetic rats 30 days after diabetes induction [20]. Increasing evidence suggest that retinal neurodegeneration is likely to be an independent pathological basis of DR [21]. In diabetic patients, internal retinal thinning was checked out, but without DR or mildest DR (microaneurysm) [22]. In the present study, under the condition of $\mathrm{HG}$, investigations were carried out on the effect of BMP-2 on the biological functions of hRECs and ARPE-19 cells. The results indicate that in hREC cells, the proliferation of cells and expressions of angiogenesis and inflammatory factors were enhanced by BMP-2 partially through the Smad signaling pathway, while the expressions of angiogenesis and inflammatory cytokines were upregulated by BMP-2 entirely through the Smad signaling pathway in ARPE-19 cells. Therefore, further studies on the molecular mechanism of retinal neurodegeneration and the signal mechanism related to BMP-2 will be very valuable in the development of early treatment strategy for DR.

The application of corticosteroids seems more significant in the treatment of DME, particularly in patients with refractory DME and more serious adverse reactions of anti-VEGF treatment. As effective anti-inflammatory drugs, corticosteroids target a wide range of mediators involved in the pathogenesis of DME. These include VEGF, TNF- $\alpha$, chemokine, leukocyte stagnation and phosphorylated tight junction protein. Unresponsiveness to anti-VEGF is mostly likely the result of a series of cytokines in DME cases. Corticosteroids are very efficacious antiinflammatory drugs which target a wide range of mediators participating in the pathogenesis of DME, such as VEGF, TNF-a, leukocyte stasis and phosphorylated human occludin proteins $[23,24]$. Interestingly, vitreous implants may induce dislocation of the anterior chamber, but this could be treated by injecting the implant with balanced saline solution [25].

Intravitreal corticosteroid therapy is effective for DME. In particular, lower frequency intraocular injections and sustained release of corticosteroids reduce costs and achieve better patient compliance. However, since the effectiveness of corticosteroid therapy for PDR has not been defined, it should be applied as first-line therapy only when anti-VEGF drugs present a high risk [26]. At the same time, as one of the most important pro-inflammatory cytokines in the vitreous tissue of DR patients, IL- 6 has been studied as a prospective target for the antiinflammatory treatment of DR [27].

\section{CONCLUSION}

The results obtained in this study indicate that BMP-2 promotes angiogenesis and inflammatory cytokine expressions via a mechanism that partially depends on the Smad signaling pathway. These findings have beneficial potentials in the management of DR.

\section{DECLARATIONS}

\section{Acknowledgement}

This work was supported by National Key R\&D Program of China (Grant nos. 2017YFA0105000 and 2018YFA0107304); Plan For Scientific Innovation Talent of Henan Province (Grant no. 184200510005), and Henan Natural Science Foundation Project (Grant no. 182300410363).

\section{Conflict of interest}

No conflict of interest is associated with this work.

\section{Contribution of authors}

We declare that this work was done by the authors named in this article, and all liabilities pertaining to claims relating to the content of this article will be borne by the authors. All authors read and approved the manuscript for publication. Zaohe Sun and Guangming Wan conceived and designed the study. Shenzhi Liang and Cheng Qian collected and analyzed the data, while Zaohe Sun wrote the manuscript.

\section{Open Access}

This is an Open Access article that uses a funding model which does not charge readers or their institutions for access and distributed under the terms of the Creative Commons Attribution License (http://creativecommons.org/licenses/by/ 4.0) and the Budapest Open Access Initiative (http://www.budapestopenaccessinitiative.org/rea d), which permit unrestricted use, distribution, 
and reproduction in any medium, provided the original work is properly credited.

\section{REFERENCES}

1. Lv J, Chen MM, Mu ZH, Wang F, Qian ZY, Zhou L, Guo $Q T$, Zhao $Z M$, Pan $Y P$, Liao $X Y$, et al. Intravitreal Bevacizumab Injection Attenuates Diabetic Retinopathy in Adult Rats with Experimentally Induced Diabetes in the Early Stage. J Diabetes Res 2018; 2018: 9216791.

2. Yang $N$, Zhang $W$, He T, Xing Y. Suppression of Retinal Neovascularization by Inhibition of Galectin-1 in a Murine Model of Oxygen-Induced Retinopathy. Journal of Ophthalmology 2017; 2017: 5053035.

3. Le YZ. VEGF production and signaling in Müller glia are critical to modulating vascular function and neuronal integrity in diabetic retinopathy and hypoxic retinal vascular diseases. Vision Res 2017; 139: 108-114.

4. Ola R, Künzel SH, Zhang F, Genet G, Chakraborty R, Pibouin-Fragner L, Martin $K$, Sessa $W$, Dubrac $A$, Eichmann A. SMAD4 Prevents Flow Induced ArterialVenous Malformations by Inhibiting Casein Kinase 2. Circulation 2018; 138(21): 2379-2394.

5. Hussein KA, Choksi K, Akeel S, Ahmad S, Megyerdi S, El-Sherbiny M, Nawaz M, Abu El-Asrar A, Al-Shabrawey M. Bone morphogenetic protein 2: a potential new player in the pathogenesis of diabetic retinopathy. Exp Eye Res 2014; 125: 79-88.

6. Xiao $F$, Wang $C$, Wang $C$, Gao $Y$, Zhang $X$, Chen $X$. BMPER Enhances Bone Formation by Promoting the Osteogenesis-Angiogenesis Coupling Process in Mesenchymal Stem Cells.Cell Physiol Biochem 2018; 45(5): 1927-1939.

7. Huang RL, Sun $Y$, Ho CK, Liu K, Tang $Q Q$, Xie $Y$, Li Q.. IL-6 potentiates BMP-2-induced osteogenesis and adipogenesis via two different BMPR1A-mediated pathways. Cell Death Dis 2018; 9(2): 144-159.

8. Liu J, An N, Ma C, Li X, Zhang J, Zhu W, Zhang Y, Li J. Correlation analysis of intestinal flora with hypertension. Exp Ther Med 2018; 16(3):2325-2330.

9. Ferreira PEB, Beraldi EJ, Borges SC, Natali MRM, Buttow NC. Resveratrol promotes neuroprotection and attenuates oxidative and nitrosative stress in the small intestine in diabetic rats. Biomed Pharmacother 2018; 105: 724-733.

10. Zhao JS, Jin HX, Gao JL, Pu C, Zhang P, Huang JJ, Cheng L, Feng G. Serum Extracellular Superoxide Dismutase Is Associated with Diabetic Retinopathy Stage in Chinese Patients with Type 2 Diabetes Mellitus. Dis Markers 2018; 2018: 8721379.

11. Stewart MW. Treatment of diabetic retinopathy: Recent advances and unresolved challenges. World J Diabetes 2016; 7(16): 333-341.

12. Yang Z, Zhu T, Mondello S, Akel M, Wong AT, Kothari $I M$, Lin F, Shear DA, Gilsdorf JS, Leung LY, et al. Serum-based phospho-neurofilament-heavy protein as theranostic biomarker in three models of traumatic brain injury: an Operation Brain Trauma Therapy (OBTT) study. J Neurotrauma 2019; 36(2): 348-359.

13. Ding $X$, Zhang M, Gu R, Xu G, Wu H. Activated microglia induce the production of reactive oxygen species and promote apoptosis of co-cultured retinal microvascular pericytes. Graefes Arch Clin Exp Ophthalmol 2017; 255(4): 777-788.

14. Zhang $X$, Chen $X, W u$ D, Liu W, Wang J, Feng Z, Cai G, Fu B, Hong $Q, D u$ J. Downregulation of connexin 43 expression by high glucose induces senescence in glomerular mesangial cells. J Am Soc Nephrol 2006; 17(6): 1532-1542.

15. Durham JT, Herman IM. Microvascular modifications in diabetic retinopathy. Curr Diab Rep 2011; 11(4): 253264.

16. Hou S, Sun X, Dong X, Lin H, Tang L, Xue M, Zhong G. Chlamydial plasmid-encoded virulence factor Pgp3 interacts with human cathelicidin peptide LL-37 to modulate immune response Microbes Infect 2019; 21(1): 50-55.

17. Mohamed HG, Idris SB, Ahmed MF, Åstrøm AN, Mustafa $K$, Ibrahim SO, Mustafa M. Influence of type 2 diabetes on local production of inflammatory molecules in adults with and without chronic periodontitis: a cross-sectional study. BMC Oral Health, 2015; 15(1): 86-95.

18. Feng $S, Y u H, Y u Y$, Geng Y, Li D, Yang C, Lv Q, Lu L, Liu T, Li G, et al. Levels of Inflammatory Cytokines IL$1 \beta, I L-6, I L-8, I L-17 A$, and TNF- $\alpha$ in Aqueous Humour of Patients with Diabetic Retinopathy. J Diabetes Res 2018; 2018: 8546423.

19. Wang W, Lo ACY. Diabetic Retinopathy: Pathophysiology and Treatments. Int J Mol Sci 2018; 19(6): 1816-1830.

20. Jiang H, Du J, Song J, Li Y, Wu M, Zhou J, Wu S. Lossof-function mutation of serine racemase attenuates retinal ganglion cell loss in diabetic mice.Exp Eye Res 2018; 175: 90-97.

21. Cheng Y, Du Y, Liu H, Tang J, Veenstra A, Kern TS. Photobiomodulation Inhibits Long-term Structural and Functional Lesions of Diabetic Retinopathy. Diabetes 2017; 67(2): 291-298.

22. He $X$, Sun D, Chen S, Xu H. Activation of liver $X$ receptor delayed the retinal degeneration of rd1 mice through modulation of the immunological function of glia. Oncotarget 2017; 8(19): 32068-32082.

23. Shen W, Lee S, Araujo J, Chung SH, Zhu L, Gillies MC. Effect of glucocorticoids on neuronal and vascular pathology in a transgenic model of selective Muller cell ablation. Glia 2014; 62(7): 1110-1124.

24. Zhu L, Zhao S, Liu S, Liu Q, Li F, Hao J. PTEN Regulates Renal Extracellular Matrix Deposit via Increased CTGF in Diabetes Mellitus. J Cell Biochem 2016; 117(5): 11871198.

25. Sousa LMMC, Campos DB, Fonseca VU, Viau P, Kfoury $J R$ Jr, Oliveira CA, Binelli M, Buratini J Jr, Papa PC. Vascular endothelial growth factor $A$ (VEGFA) modulates bovine placenta steroidogenesis in vitro. Placenta 2012; 33(10): 788-794. 
26. Obeid A, Gao X, Ali FS, Talcott KE, Aderman CM, Hyman L, Ho AC, Hsu J. Loss to Follow-Up in Patients with Proliferative Diabetic Retinopathy after Panretinal Photocoagulation or Intravitreal Anti-VEGF Injections. Ophthalmology 2018; 125(9): 1386-1392.
27. González-Chávez MM, Alonso-Castro AJ, ZapataMorales JR, Arana-Argáez V, Torres-Romero JC, Medina-Rivera YE, Sánchez-Mendoza E, PérezGutiérrez S. Anti-inflammatory and antinociceptive effects of tilifodiolide, isolated from Salvia tiliifolia Vahl (Lamiaceae). Drug Dev Res 2018; 79(4): 165-172. 